\title{
In-silico Structure Modeling and Docking Studies Using Dipeptidyl Peptidase 4 (DPP4) Inhibitors against Diabetes Type-2
}

\author{
Rajneesh Prajapat", Ijen Bhattacharya
}

Department of Medical Biochemistry, Faculty of Medical Sciences, Rama Medical College and Hospital, Rama University, India

Copyright $\subseteq 2016$ by authors, all rights reserved. Authors agree that this article remains permanently open access under the terms of the Creative Commons Attribution License 4.0 International License

\begin{abstract}
Recently recognized class of oral hypoglycemic, dipeptidyl peptidase (DPP4) inhibitors could block the dipeptidyl peptidase 4 (DPP4) enzymes. DPP4 is an intrinsic membrane glycoprotein and a serine exopeptidase that plays a major role in glucose metabolism and responsible for the degradation of incretins such as GLP-1, therefore providing a useful treatment to diabetes mellitus type 2 . The present work focused on the study of the structural homology modeling of dipeptidyl peptidase 4 [Homo sapiens] (NP_001926). The Ramachandran plot of DPP4 (NP_001926.2) has $88.9 \%$ residues in the most favoured region while template 2QT9 has $96.1 \%$ residues in the most favoured region. The model was validated by using protein structure tools RAMPAGE and Prochek for reliability. Docking studies were further performed to analyze the interaction mode between selected DPP4 inhibitor anagliptin derivative SKK and receptor DPP4 by using Hex 8.0.0. The in-silico analysis was useful to identify the novel inhibitor that illustrates better activity than the other reported inhibitors.
\end{abstract}

Keywords Diabetes, Docking, SKK, DPP4, In-silico Analysis

\section{Introduction}

Diabetes mellitus is a prime public health problem and forthcoming epidemic all over the globe [38] disorder caused due to insufficient or ineffective insulin [2]. In India, there were approximately 40 million people suffering from diabetes and this number possibly will rise up to 300 million by 2025 [45; 46]. According to International Diabetes Federation record, worldwide the number of people with diabetes will be increase from current figure of 240 million to 380 million over the next 20 years $[11 ; 37]$ and up to 642 million by 2040 [10].

The combination therapeutics or oral monotherapy with other anti-diabetic agents used to control diabetes through clinical anti-diabetes therapy [16]. But, these anti-diabetic agents may cause adverse side effects and chronic complications $[6 ; 25]$.

Dipeptidyl peptidase-4 (DPP4) encoded by the DPP4 gene and it is a membrane serine exopeptidase that cleaves $\mathrm{X}$-proline dipeptides from the N-terminus of polypeptides $[12 ; 40]$. DPP-4 inhibitors recommended for use in patients with T2DM therapy, that including hypoglycaemia incidences, risks of cardiovascular complications and weight gain [14]. It is a rather indiscriminate enzyme for which a diverse range of substrates are known. Dipeptidyl peptidase 4 (DPP4) is a serine protease that [36] that degrade peptidic hormone glucagon-like peptide 1 (GLP-1). GLP-1 plays an important role in the regulation of insulin release to control the level of blood sugar in human body [18]. Several studies demonstrated that the inhibition of DPP4 could increase the amount of circulating GLP-1 to improve the secretion of insulin in the body [43]. Therefore, it has regarded as promising target to develop novel drug for treatment of type 2 diabetes.

So far, a couple of identified DPP4 inhibitors, such as sitagliptin and saxagliptin, were approved to be used clinically as antidiabetic drugs by FDA [41; 20]. However, there is still a need for more potent, selective and safer DPP4 inhibitor, which does not have the inspecificity and side effect possessed by the presently available inhibitors [13], because of worldwide problem of type 2 diabetes. The recent research on DPP4 inhibitors using in silico methods is focus on physicochemical properties analysis[3].Therefore, researcher are studying new DPP4 ligands for the development of the novel anti-diabetes drug.

Homology modelling provides structural information about the protein dynamics, function, interactions with other proteins and ligands $[31 ; 7]$. In the present study, homology modelling [22] process was used to determine the structure of dipeptidyl peptidase 4 [Homo sapiens] (NP_001926).

The docking was performed to reveal the interaction mode and to predict the orientation $[29 ; 30]$ between inhibitors 
SKK and DPP4 by using Hex 8.0.0. The docking outcome demonstrated the binding conformation of anagliptin derivative SKK and DPP4. The results of the study could be used to design or predict new potent DPP4 inhibitors. In silico analysis is an effective way to develop the anti-diabetic drug model.

\section{Material and Methods}

\subsection{Sequences Retrieval and Alignment}

The amino acid sequence of dipeptidyl peptidase 4 [Homo sapiens] (NP_001926) was retrieved from NCBI (www.ncbi.nlm.nih.gov). Multiple sequence alignment was performed by using BLASTp [1; 44] against the PDB (Protein Databank), to find out the related homologues. The PDB file of DPP4 (NP_001926) was generated by using 3D-JIGSAW. The PDB file of homologous template and query were further utilized for 3D model energy validation [17].

\subsection{Molecular Modelling of DPP4}

The UCLA-DOE provide an analysis about the quality of a putative crystal structure for protein. RAMPAGE program was used to visualize and evaluate the Ramachandran plot of DPP4 (NP_001926) and its homologous template [23]. The models were analyzed based on various factors such as G-factor, number of residues in core, generously allowed and disallowed regions in Ramachandran plot. The validation of structure models was performed by using PROCHECK [21]. QMEAN [8] and ProSA [42] were further used for the analysis of model. The ProSA server displays the Z-score and energy plots.

The 3D structure selected as a template for constructing the model for DPP4 (NP_001926) was Human dipeptidyl peptidase (PDB access code: 2QT9). 2QT9 has 766 amino acid residues and a resolution of $2.1 \AA$ [19]. 2QT9 was illustrated $99 \%$ identity with the target sequence (2QT9).
The quality of folding was checked by using Verify3D. The system energy and 3D alignment were monitored using Atomic Non-Local Environment Assessment (ANOLEA) [24]. Produced models of homologous template and query were ranked on QMEAN server and utilized for the ribbon structural model construction. QMEAN locate the position of active site amino acids of protein and estimates the per-residue error [9].

\subsection{Docking of DPP4 (NP_001926) with SKK}

The aim of protein docking is to determine 3D structure of a protein complex preliminary from its unbound components [15]. Docking was performed to analyze the interaction between selected DPP4 inhibitor anagliptin derivative SKK [Synonyms: Anagliptin, $\mathrm{C}_{19} \mathrm{H}_{25} \mathrm{~N}_{7} \mathrm{O}_{2}$ ] (Figure 1) and receptor DPP4 (NP_001926) by using Hex 8.0.0 [34]. The PDB files of DPP4 (NP_001926) and SKK was used as inputs for the protein-protein docking. Hex 8.0.0 is a docking program; that performed structural refinement, energy minimization and display the fast 3D superposition using the SPF correlation approach. Based on the energy minimization the best front of the docked complex was selected [35].

\section{Results and Discussion}

\subsection{Building of Protein Model}

The sequence alignment of dipeptidyl peptidase 4 [Homo sapiens] (NP_001926) revealed the sequence homology with human dipeptidyl peptidase (PDB access code: 2QT9), which has 766 amino acid residues and a resolution of $2.1 \AA$ [19]. The 2QT9 (with 99\% sequence identity) was selected as template for the model building of DPP4 (NP 001926) protein. Total 766 residues were modeled with 99\% confidence by the single highest scoring template. To build the model, BLAST was run with the maximum E-value allowed for template being 0.0 .

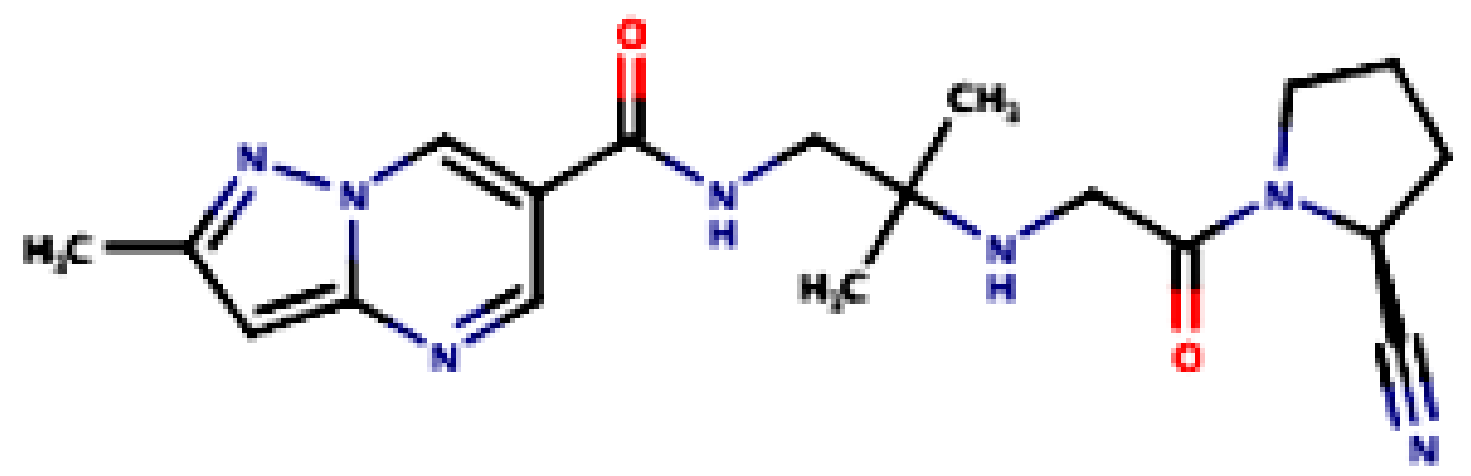

Figure 1. SKK (N-[2-(\{2-[(2S)-2-cyanopyrrolidin-1-yl]-2-oxoethyl $\}$ amino)-2-methylpropyl]-2-methylpyrazolo[1,5-a]pyrimidine-6-carboxamide) 


\subsection{Model Reputation}

The DPP4 (NP_001926) model was illustrated good stereo-chemical property in terms of overall G-factor value of -0.73. The probability conformation with $88.9 \%$ residues in the favoured region of Ramachandran plot, illustrated high accuracy of model predicted [32]. The residues number in allowed and outlier region of plot were $8.0 \%$ and $19 \%$ (Figure 2). The Ramachandran plot of template (2QT9) has $96.1 \%$ residues in favoured region, $3.9 \%$ in allowed region and $0.1 \%$ in outlier regions. (Figure 3, Table 1).

Table 1. Results summary of the Ramachandran plot

\begin{tabular}{|c|c|c|c|c|c|}
\hline \multirow{2}{*}{ Accession No } & \multirow{2}{*}{ Protein } & \multirow{2}{*}{ Description } & \multicolumn{3}{|c|}{ Residues (\%) } \\
\cline { 4 - 6 } & & & Favoured regions & Allowed regions & Outlier regions \\
\hline NP_001926.2 & DPP4 & Homo sapiens & 88.9 & 8.0 & $19 \%$ \\
\hline 2QT9 & DPP IvCD26 & Homo sapiens & 96.1 & 3.9 & 0.1 \\
\hline
\end{tabular}
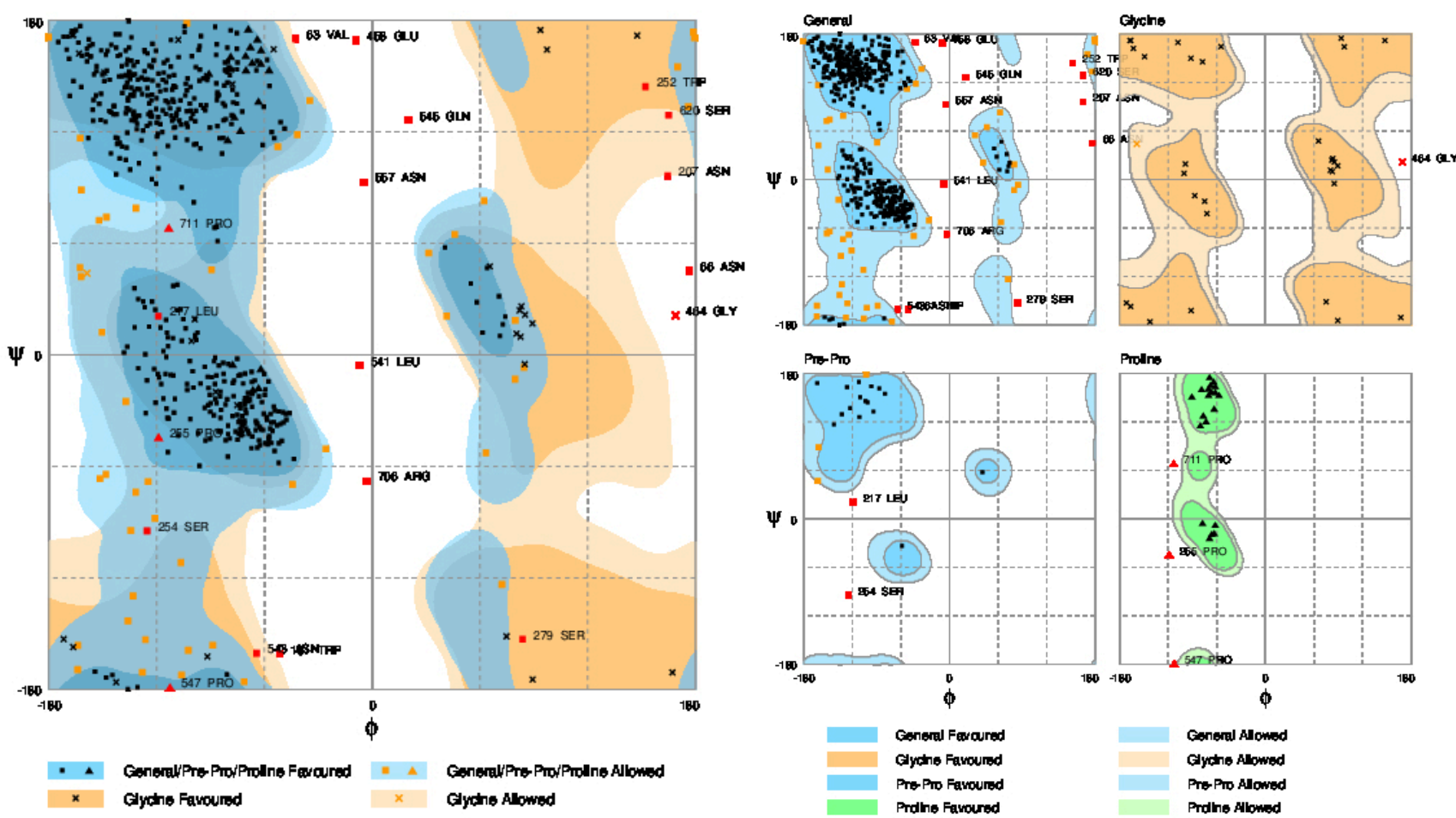

Figure 2. Ramachandran Plot analysis of dipeptidyl peptidase 4 (NP_001926.2) [Homo sapiens] protein. (a) Total number of residues were 601 with $88.9 \%$ in most favored regions [A, B, L], $8.0 \%$ in allowed regions [a,b,l,p], and $19 \%$ in outlier region regions. (b) Non-proline residues and non-glycine residue regions. 

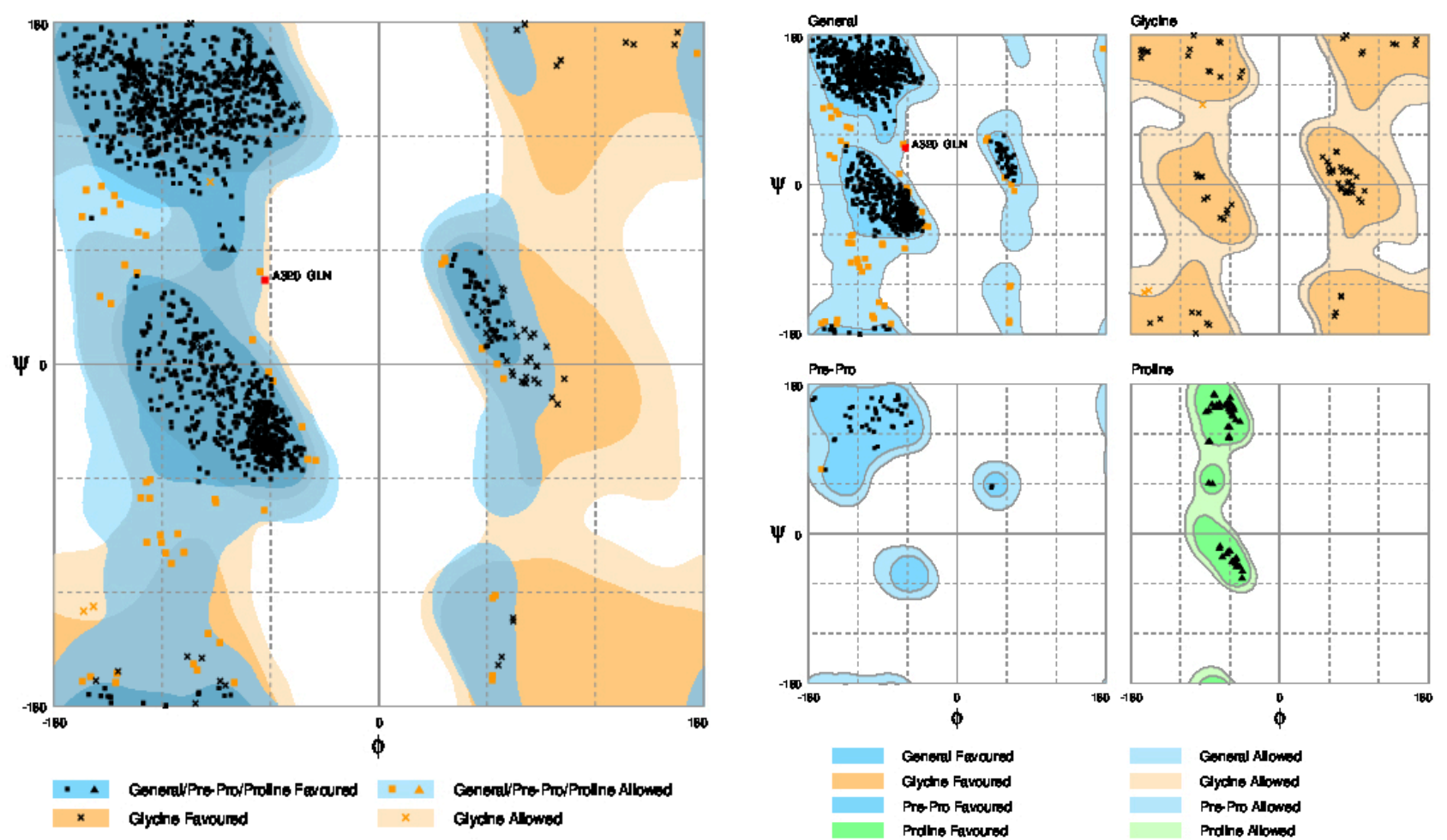

Figure 3. Ramachandran Plot analysis of homologous Human Dipeptidyl Peptidase IvCD26 in complex with a 4-Aryl Cyclohexylalanine Inhibitor (2QT9) protein. (a) Total number ofresidues were 1452 with $96.1 \%$ in most favored regions [A, B, L], $3.9 \%$ in allowed regions [a,b,l,p], and $0.1 \%$ in outlier region regions. (b) Non-proline residues and non-glycine residue regions

The Ramachandran plot of DPP4 (NP_001926) has only 88.9\% residues in the most favoured region and its template 2QT9 [Human dipeptidyl peptidase] has higher $96.1 \%$ residues in most favoured regions, hence 2QT9 is more stable than DPP4 (NP_001926) (Table 1). A good quality Ramachandran plot has more than $90 \%$ in the most favoured regions [26] thus the 2QT9 model could be include in good quality category. For target DPP4 (NP_001926) model, energy minimization should be required to enhance stability by using application of simulated annealing, steepest descent and conjugate gradient.

\subsection{Model Validation}

The problems of protein structures based on energy plots could be easily seen by ProSA and displayed in a three-dimensional manner. ProSA web was used to check the three dimensional model errors of DPP4 (NP_001926) and 2QT9 (Figure 4 and Figure 5). ProSA web z-scores of protein chains were determined by X-ray crystallography (light blue) or NMR spectroscopy (dark blue) with respect to their length. The z-scores of DPP4 (NP_001926) and DPP IvCD26 (2QT9) is highlighted as large dots. 

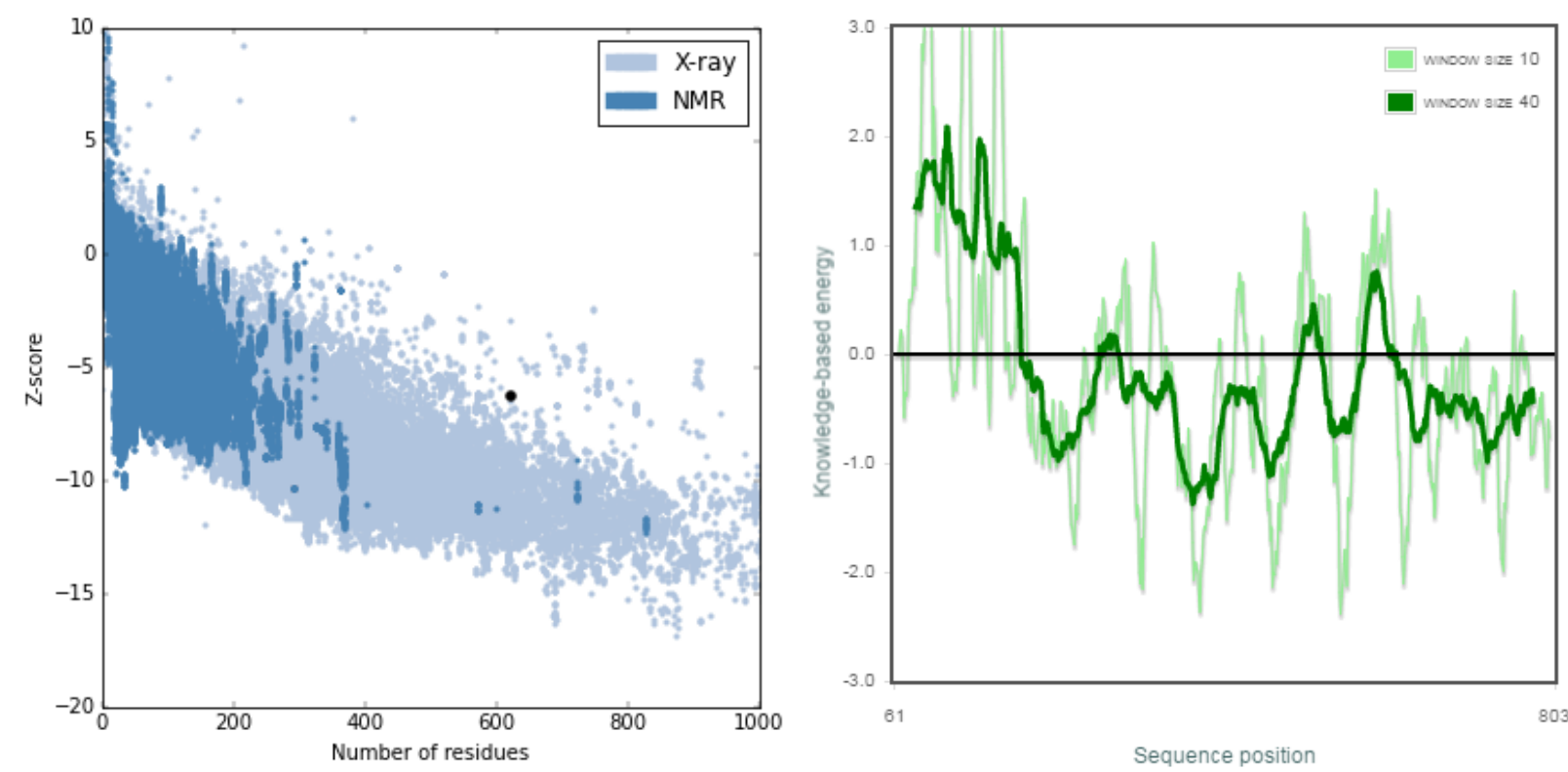

Figure 4. ProSA web service analysis of DPP4 (NP_001926) [Homo sapiens] protein overall model quality (a) and local model quality (b).
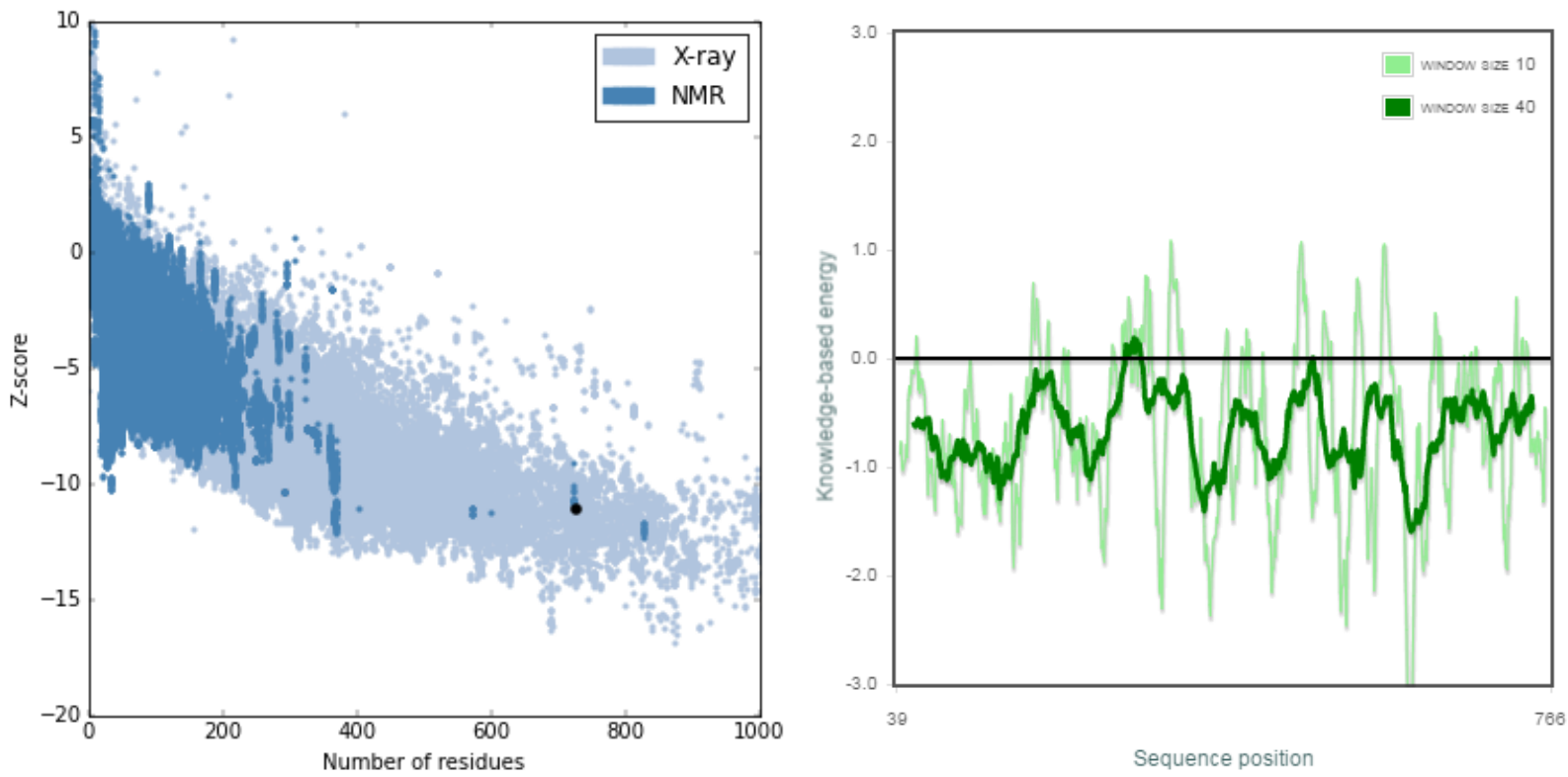

Figure 5. ProSA web service analysis of DPP IvCD26 (2QT9) [Homo sapiens] protein overall model quality (a) and local model quality (b).

The ProSA z-score was -6.21 for DPP4 (NP_001926) [Figure 4] and -11.09 for the DPP IvCD26 (2QT9) protein, indicates the overall model quality of target and template (Figure 5) measures the deviation of the total energy of the structure with respect to an energy distribution [39]. 


\section{Density plot for QMEAN scores of the reference set}

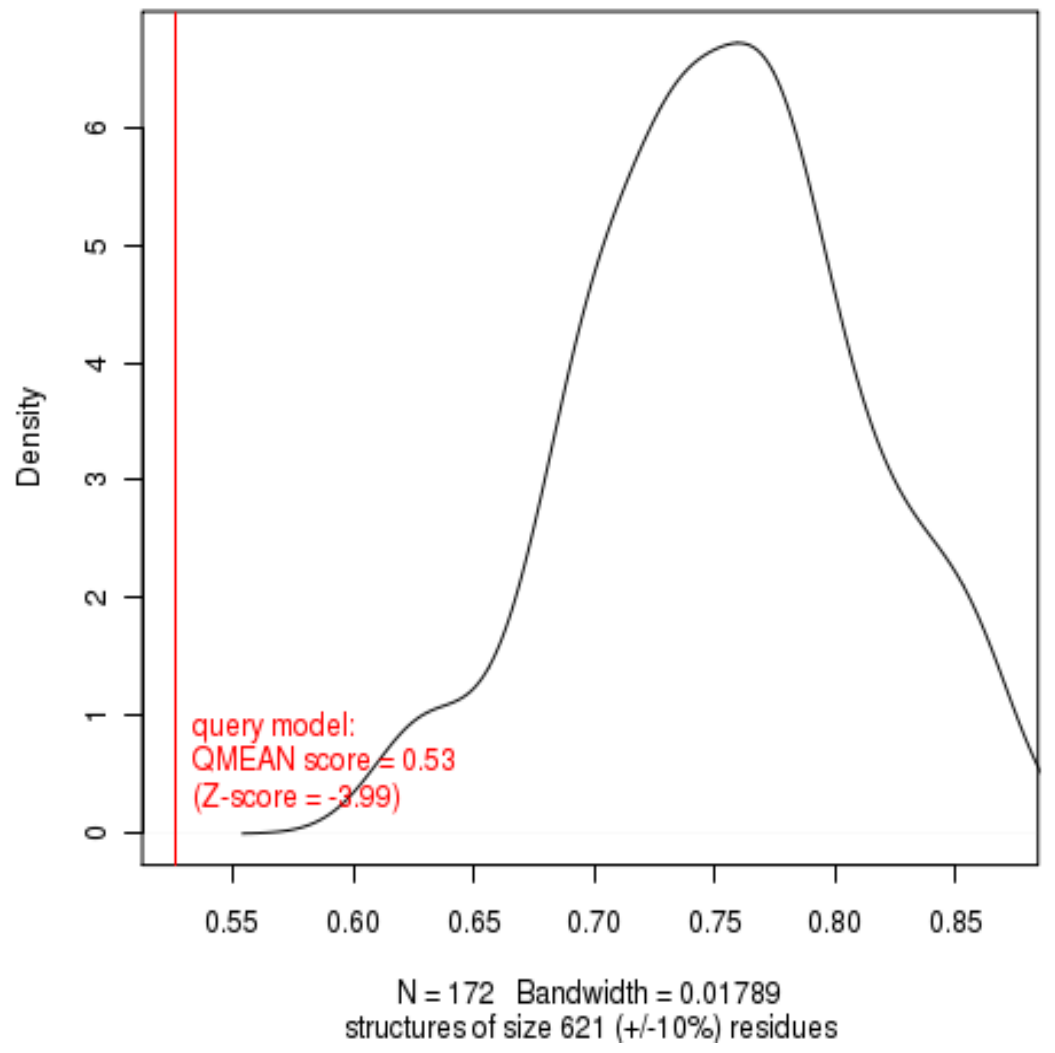

Figure 6a. The density plot for target DPP4 (NP_001926) [Homo sapiens] showing the value of Z-score and QMEAN score

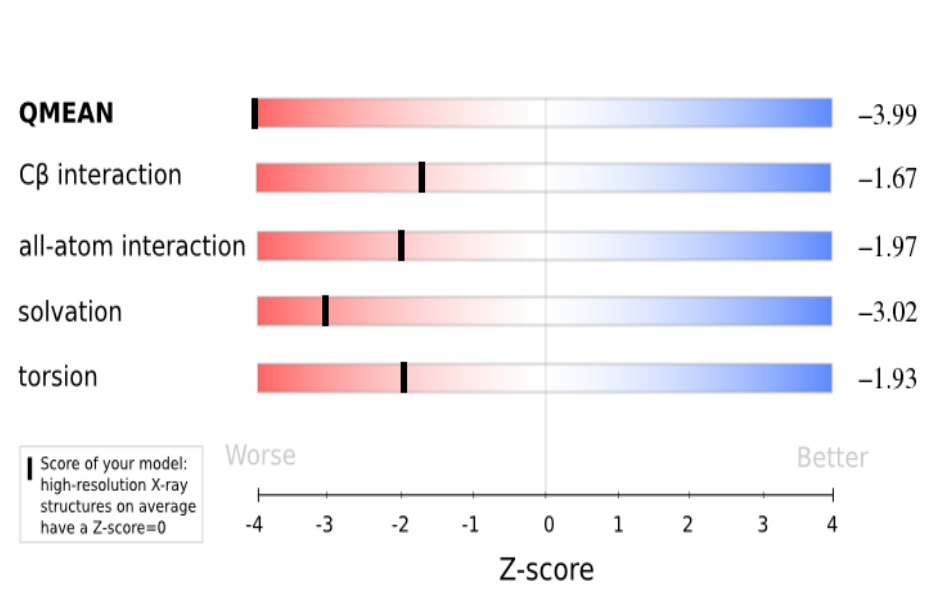

Comparison with non-redundant set of PDB structures

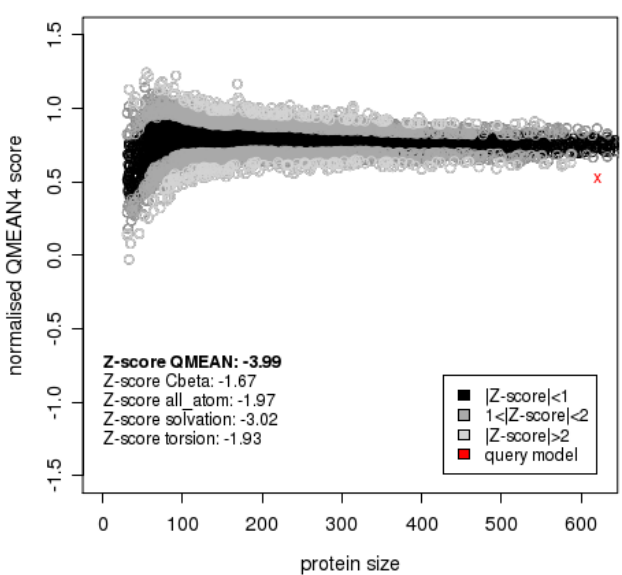

Figure 6b. Plot showing the QMEAN value as well as Z-score (for NP_001926) 
Density plot for QMEAN scores of the reference set

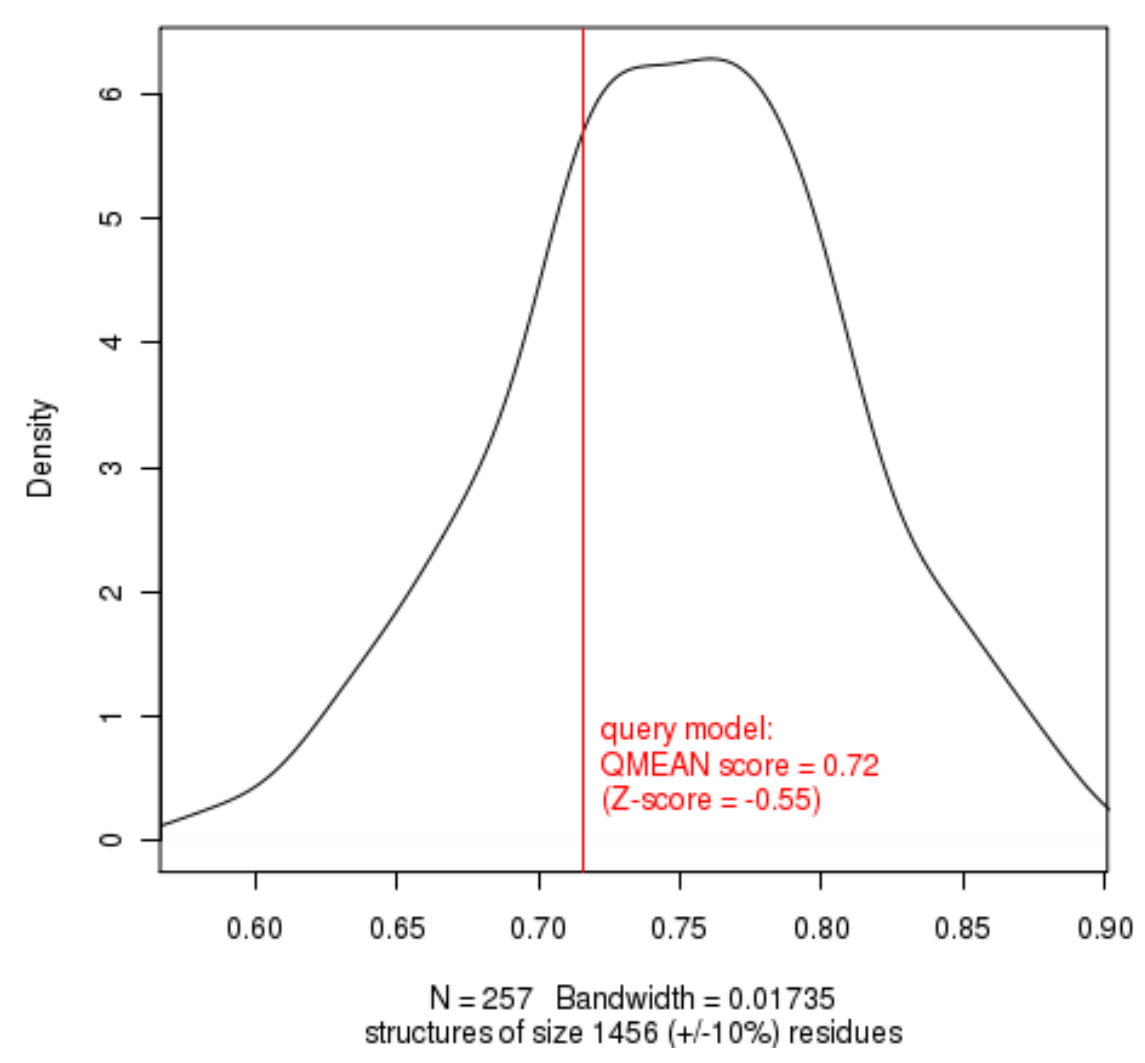

Figure 7a. The density plot for target DPP IvCD26 (2QT9) [Homo sapiens] showing the value of Z-score and QMEAN score
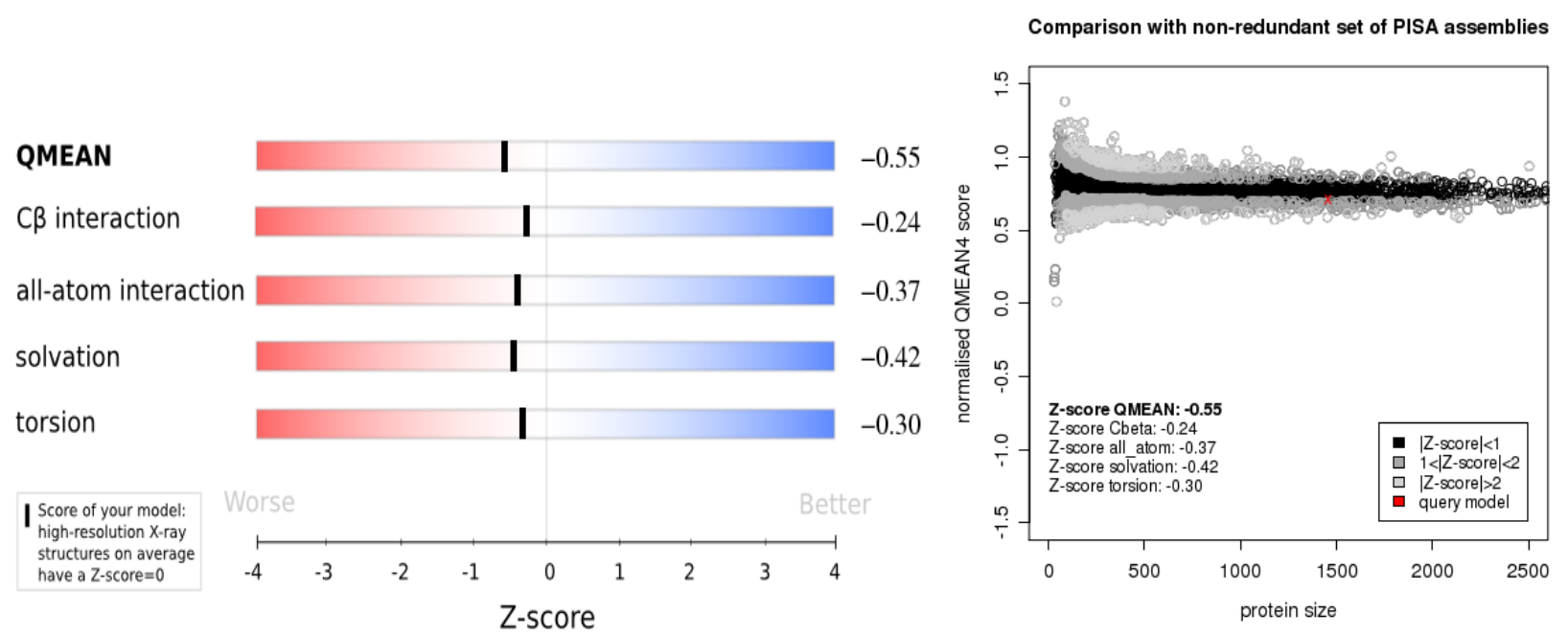

Figure 7b. Plot showing the QMEAN value as well as Z-score (for 2QT9)

QMEAN score of target DPP4 (NP_001926) model was 0.53 and Z-score was -3.99 (Figure 6a). QMEAN score of template DPP IvCD26 (2QT9) was 0.72 with Z-score was -0.55 (Figure 7a), which very closed to the value of 0 . That illustrated the fine quality of the both models [42]. The reliability of the model was expected in between 0 and 1 and this could be inferred from the density plot for QMEAN scores of the reference set. A comparison between protein size and normalized QMEAN score (0.40) in non-redundant set of PDB structures plot revealed different set of z-values for different parameters such as C-beta interactions (-1.67), interactions between all atoms (-1.97), solvation (-3.02) and torsion (-1.93) [Figure 6b]. The ribbon model of DPP4 (NP_001926) and 2QT9 was generated using QMEAN server (Figure 8 and Figure 9). The per-residue error visualized using a color gradient from blue (more reliable region) to red (potentially unreliable region). 


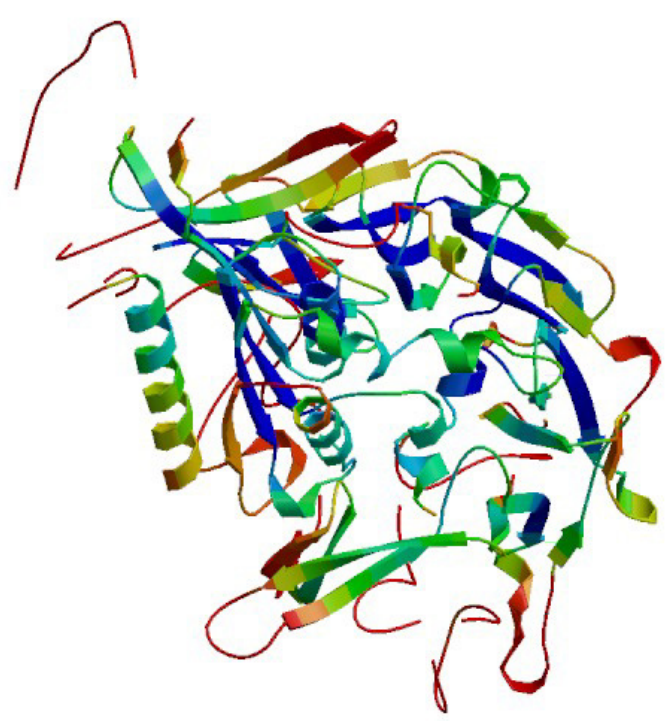

Figure 8. DPP4 (NP_001926) [Homo sapiens] ribbon model generated using QMEAN server. Estimated per-residue error visualized using a color gradient from blue (more reliable region) to red (potentially unreliable region).

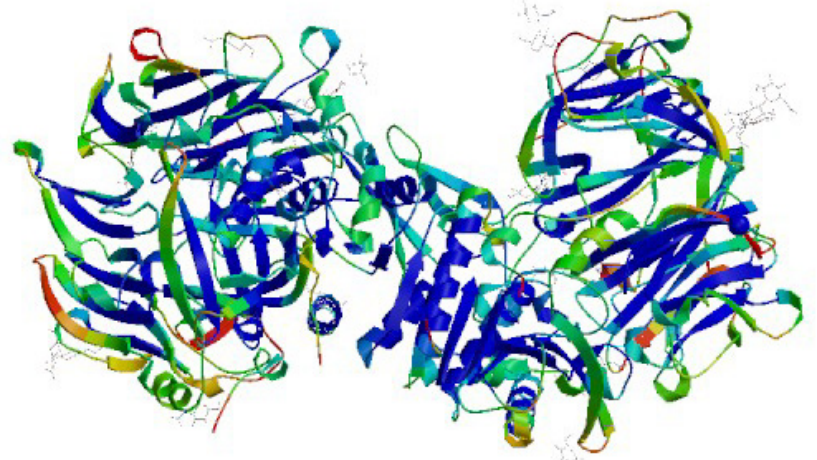

Figure 9. DPP IvCD26 (2QT9) [Homo sapiens] ribbon model generated using QMEAN server. Estimated per-residue error visualized using a color gradient from blue (more reliable region) to red (potentially unreliable region).

Protein docking predicts symmetrical 3D models of protein-protein complexes with arbitrary point group symmetry [33]. Hex assigns multiple local coordinate systems to larger molecule (receptor DPP4 [NP_001926]) and docks the ligand (SKK) around each local coordinate frame on the receptor. Figure represents Hex view of DPP4 (NP_001926) protein domain and SKK in vander waals mode, with the intermolecular axis (10a) and solid surfaces form (10b).
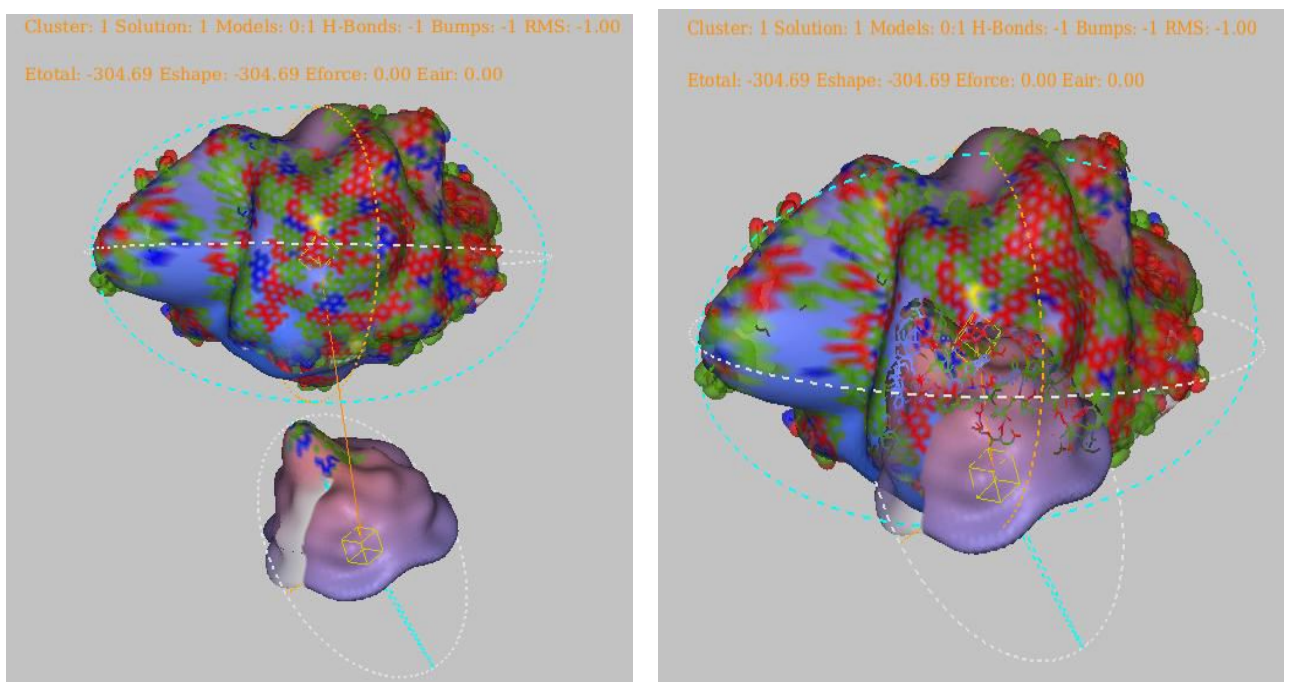

Figure 10. A Hex scene showing the DPP4 (NP_001926) domain and SKK (below) in Vander Waals mode and with the intermolecular axis drawn in blue (a) and solid surfaces (b) 
On the basis of the RMS and energy values, the best docking orientation was selected. The better RMS value of docking was -1.00.The binding sites of protein reveal chemical specificity, to determine the nature of ligand and affinity strength of chemical bond [4,5]. The 1 Clst initial Etotal, Eshape and Eforce values for the model were -304.7, -304.7 and 0.0 (Table 2).

Table 2. Clustering found 560 clusters from 2000 docking solutions in 0.05 seconds.

\begin{tabular}{|c|c|c|c|c|c|c|c|c|}
\hline Clst & Soln & Models & E-total & E-shape & E-force & E-air & Bmp & RMS \\
\hline 1 & 1 & $000: 001$ & -304.7 & -304.7 & 0.00 & 0.0 & -1 & -1.00 \\
\hline 1 & 2 & $000: 001$ & -304.6 & -304.6 & 0.00 & 0.0 & -1 & -1.00 \\
\hline 1 & 7 & $000: 001$ & -295.7 & -295.7 & 0.00 & 0.0 & -1 & -1.00 \\
\hline 1 & 8 & $000: 001$ & -294.7 & -295.7 & 0.00 & 0.0 & -1 & -1.00 \\
\hline 1 & 9 & $000: 002$ & -292.7 & -292.7 & 0.00 & 0.0 & -1 & -1.00 \\
\hline 1 & 11 & $000: 001$ & -288.3 & -288.3 & 0.00 & 0.0 & -1 & -1.00 \\
\hline 1 & 12 & $000: 002$ & -286.3 & -286.3 & 0.00 & 0.0 & -1 & -1.00 \\
\hline 1 & 21 & $000: 002$ & -282.4 & -282.4 & 0.00 & 0.0 & -1 & -1.00 \\
\hline 1 & 23 & $000: 001$ & -280.5 & -280.5 & 0.00 & 0.0 & -1 & -1.00 \\
\hline 1 & 28 & $000: 002$ & -277.7 & -277.7 & 0.00 & 0.0 & -1 & -1.00 \\
\hline 1 & 30 & $000: 001$ & -276.0 & -276.0 & 0.00 & 0.0 & -1 & -1.00 \\
\hline 1 & 37 & $000: 001$ & -272.6 & -272.6 & 0.00 & 0.0 & -1 & -1.00 \\
\hline 1 & 43 & $000: 002$ & -270.8 & -270.8 & 0.00 & 0.0 & -1 & -1.00 \\
\hline 1 & 44 & $000: 002$ & -270.6 & -270.6 & 0.00 & 0.0 & -1 & -1.00 \\
\hline 1 & 45 & $000: 001$ & -270.4 & -270.4 & 0.00 & 0.0 & -1 & -1.00 \\
\hline 1 & 49 & $000: 001$ & -270.2 & -270.2 & 0.00 & 0.0 & -1 & -1.00 \\
\hline 1 & 54 & $000: 001$ & -268.8 & -268.8 & 0.00 & 0.0 & -1 & -1.00 \\
\hline 1 & 65 & $000: 001$ & -265.7 & -265.7 & 0.00 & 0.0 & -1 & -1.00 \\
\hline 1 & 78 & $000: 001$ & -263.4 & -263.4 & 0.00 & 0.0 & -1 & -1.00 \\
\hline 1 & 87 & $000: 001$ & -261.6 & -261.6 & 0.00 & 0.0 & -1 & -1.00 \\
\hline
\end{tabular}

Figure illustrates spherical harmonic surfaces to order L $=12$ for the DPP4 (NP_001926) protein domain and SKK (Figure 11). Illustration of the DPP4/SKK complex shown as contoured Gaussian density surfaces and background modes (Figure 11). Figure illustrates docking control results in the form of solid models (Figure 12a) and solid surface (Figure 12b) model view of DPP4 (NP_001926) and SKK complex. These docking results suggest that SKK interacts with the DPP4 protein of and causes its inhibition [28]. The binding pocket values for DPP4 model were predicted by using Hex 8.0.0. The predicted two pockets by the software with different primary surface area and volume shown in Table 3 .
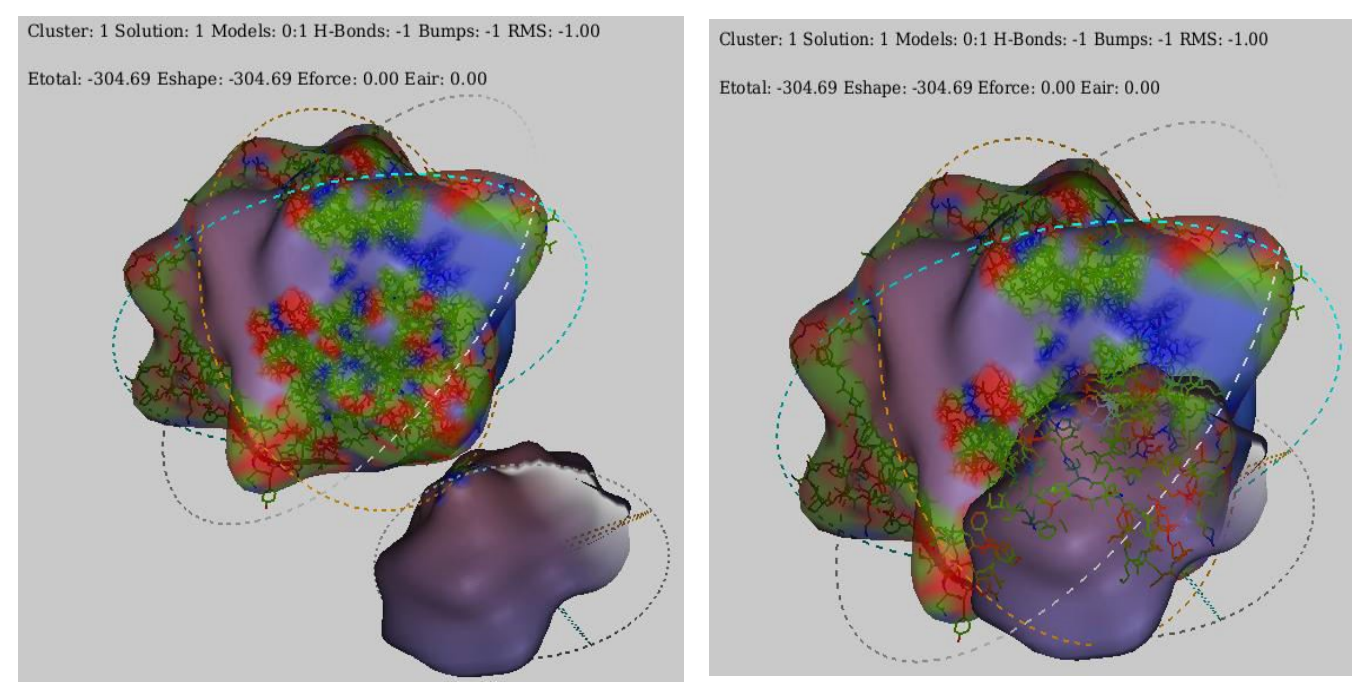

Figure 11. Illustration of spherical harmonic surfaces to order L $=12$ for the DPP4 (NP 001926) domain and SKK (a). Illustration of the DPP4 (NP_001926) (Receptor) and SKK (Ligand) complex shown as contoured Gaussian density surfaces and coloured by chain colour, drawn using perspective (keyboard P) and background (keyboard B) modes (b) 

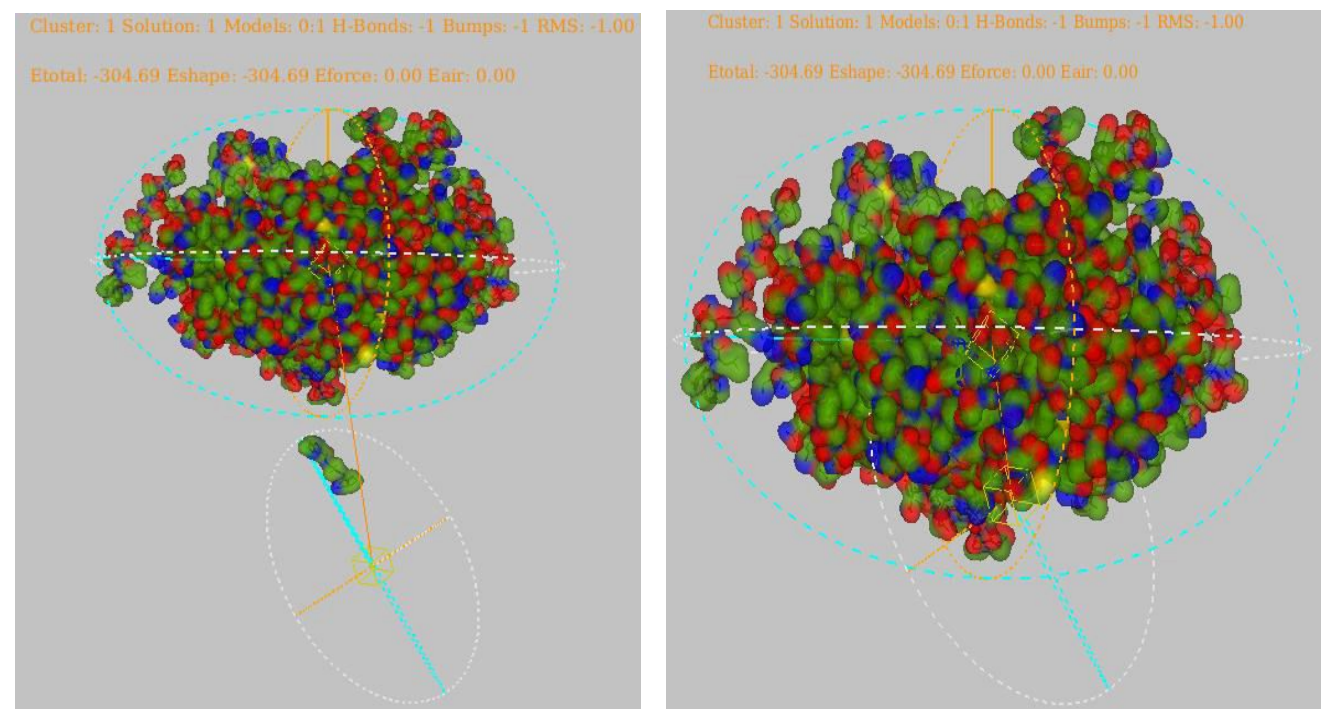

Figure 12. Docking results illustrate solid models (a) and solid surface (b) view forms of DPP4 (NP_001926) /SKK complex

Table 3. Binding site model DPP4 (NP_001926) protein. Calculated 29 surface normals (docking orientations) in 0.28 seconds. Calculating canonical orientation to $\mathrm{L}=12$

\begin{tabular}{|c|c|c|c|c|c|c|c|c|c|c|c|}
\hline \multirow{2}{*}{ Pocket } & \multirow{2}{*}{$\begin{array}{l}\text { Polar } \\
\text { Probe }\end{array}$} & \multirow{2}{*}{$\begin{array}{l}\text { Apolar } \\
\text { probe }\end{array}$} & \multirow{2}{*}{$\begin{array}{c}\text { Primary } \\
\text { Surface } \\
\text { area }\end{array}$} & \multirow{2}{*}{$\begin{array}{l}\text { Primary } \\
\text { Surface } \\
\text { volume }\end{array}$} & \multirow{2}{*}{$\begin{array}{c}\text { Typical } \\
\text { Edge arc }\end{array}$} & \multirow{2}{*}{$\begin{array}{c}\text { Typical } \\
\text { edge length } \\
(\AA)\end{array}$} & \multirow{2}{*}{$\begin{array}{c}\text { Average } \\
\text { radius } \\
(\AA)\end{array}$} & \multirow{2}{*}{$\begin{array}{l}\text { Surface } \\
\text { area }(\AA)\end{array}$} & \multicolumn{3}{|c|}{ Triangles } \\
\hline & & & & & & & & & Min. & Max. & Avg. \\
\hline 1 & $0.00 \mathrm{~A}$ & $0.00 \mathrm{~A}$ & 51389.81 & 62777.83 & 4.62 & 2.59 & 32.14 & 20349.10 & 1.84 & 58.99 & 4.52 \\
\hline 2 & $0.00 \mathrm{~A}$ & $0.00 \mathrm{~A}$ & 51084.10 & 66988.55 & 2.80 & 0.69 & 14.07 & 1.3433 & 0.00 & 37.04 & 1.24 \\
\hline
\end{tabular}

Docking could be used to study the mechanism of enzymatic reaction, to identify possible binding modes for a ligand and to screen a database [27]. A few amino acids were found to be conserved in DPP4 (NP_001926), that forming the binding cavity for the SKK. Interaction of SKK with DPP4 (NP_001926), used as a guideline to design and predict new potent DPP4 inhibitors, which could be an effective way to find novel leads for the development of anti-diabetic drugs. The anaglyptic derivative improves the glycemic control; hence, SKK might be consider as novel potent DPP4 inhibitor.

\section{Conclusion}

The DPP4 inhibitors accepted as new drug class because it provides effective glycemic control and low risk of hypoglycemia. In silico modeling and docking analyses illustrated that the anagliptin derivative SKK could be inhibit DPP4 enzymatic activity. The results of study could be used to design and predict new potent DPP4 inhibitors, which could be an efficient mode for designing of anti-diabetic drugs. The results will be further supportive to screen new DPP4 inhibitors from nature sources also. The in silico techniques are useful to identify the novel inhibitors for the clinical trial of diabetic patients.

\section{REFERENCES}

[1] Altschul SF, Madden TL, Schaffer AA, Zhang J, Zhang Z, Miller W, Lipman DJ. Gapped BLAST and PSI-BLAST: A new generation of protein database search programs, Nucleic Acids Res. 1997; 25 (17): 3389-3402.

[2] Awasthi A, Parween N, Singh VK, Anwar A., Prasad B, Kumar J. Diabetes: Symptoms, Cause and Potential Natural Therapeutic Methods. Adv. in Diabetes and Metabolism. 2016; 4, 10-23. doi: 10.13189/adm.2016.040102.

[3] Balajee R, Dhanarajan MS. Identification and Comparative Molecular Docking Analysis of 6, 7, 8, 9-Tetrahydro-2H-11oxa-2, 4, 10-triaza-benzo[B] fluoren-1-one (RBMS-01) Bounds with DPP4 for Anti-Hyperglycemic Activities. Chem Sci Trans. 2012, 1(2), 279-288. DOI:10.7598/cst2012.166

[4] Balakrishnan M, Srivastava RC. Protein Structural Modeling of Acetylglutamate Kinase from Leptospira Interrogans and Docking Studies of N-Acetyl-L-Glutamate. The IUP J. of Biotech. 2009; 3 (3): 7-16.

[5] Balakrishnan M., Srivastava RC, Pokhriyal M. Homology modeling and docking studies between HIV-1 protease and carbamic acid. Indian J. Biotechnol. 2010; 9: 96-100.

[6] Barnett AH. Thiazolidinediones and cardiovascular outcomes. Brit. J. Diabetes Vasc Dis. 2008; 8:45-9.

[7] Barry MM, Mol CD, Anderson WF, Lee JS. Sequencing and modeling of anti-DNA immunoglobulin $\mathrm{Fv}$ domains. Comparison with crystal structures, J. Biol Chem. 1994; 269 : 3623-3632.

[8] Benkert P, Künzli M, Schwede T. QMEAN server for protein 
model quality estimation. Nucleic Acids Res. 2009; 37: W510-4. doi: 10.1093/nar/gkp322., 2009.

[9] Benkert P, Schwede, T, Tosatto, SCE. QMEANclust: Estimation of protein model quality by combining a composite scoring function with structural density information. BMC Struct Biol. 2009; 9:35. doi: 10.1186/1472-6807-9-35.

[10] Cefalu WT, Buse JB, Tuomilehto J, Fleming GA, Ferrannini E, Gerstein HC, Bennett PH, Ramachandran A, Raz I, Rosenstock J, Kahn SE. Update and next steps for Real-World Translation of Interventions for Type 2 Diabetes Prevention: Reflections from a Diabetes Care Editors' Expert Forum. Diabetes Care. 2016; 39 (7) 1186-1201. DOI: $10.2337 / \mathrm{dc} 16-0873$.

[11] Chada RR, Sethi BK, Waghray K and Naidu SK. Obesity and Type 2 Diabetes: A Population Based Study of Urban School Children in South India. Adv. in Diabetes and Metabolism.2014; 2, 4-9. doi: 10.13189/adm.2014.020102.

[12] Darmoul D, Lacasa M, Baricault L, Marguet D, Sapin C, Trotot P, Barbat A, Trugnan G. Dipeptidyl peptidase IV (CD26) gene expression in enterocyte-like colon cancer cell lines HT-29 and Caco-2: cloning of the complete human coding sequence and changes of dipeptidyl peptidase IV mRNA levels during cell differentiation. J. Biol Chem. 1992, 267: 4824-4833.

[13] Defronzo RA, Okerson T, Viswanathan P, Guan X, Holcombe JH, MacConell L. Effects of exenatide versus sitagliptin on postprandial glucose, insulin and glucagon secretion, gastric emptying, and caloric intake: a randomized, cross-over study. Curr Med Res Opin. 2008; 24: 2943-2952.

[14] Galstyan KO, Nedosugova LV, Petunina NA, Trakhtenberg JA, Vostokova NV, Karavaeva OV, Chasovskaya TE. First Russian DPP-4 inhibitor Gosogliptin comparing to Vildagliptin in type 2 diabetes mellitus patients. Diabetes mellitus. 2015; 19(1):89-96.

[15] Ghoorah AW, Devignes MD, Smaïl-Tabbone M, Ritchie DW. Protein docking using case-based reasoning. Proteins. 2013; 81: 2150-2158. doi:10.1002/prot. 24433 .

[16] Havale SH, Pal M. Medicinal chemistry approaches to the inhibition of dipeptidyl peptidase-4 for the treatment of type 2 diabetes. Bioorg Med. Chem. 2009; 17:1783-802.

[17] Heinrichs A. Proteomics: Solving a 3D jigsaw puzzle. Nat Rev Mol Cell Biol. 2008; 9: 3-3.

[18] Jiang C, Han S, Chen T, Chen J.3D-QSAR and docking studies of arylmethylamine-based DPP IV inhibitors. Acta Pharmaceutica Sinica B. 2012; doi:10.1016/j.apsb.2012.06.0 07

[19] Kaelin DE, Smenton AL, Eiermann GJ, He H, Leiting B, Lyons KA, Patel RA, Patel SB, Petrov A, Scapin G, Wu JK, Thornberry NA, Weber AE, Duffy JL. 4-arylcyclohexylalanine analogs as potent, selective, and orally active inhibitors of dipeptidyl peptidase IV. Bioorg Med Chem Lett. 2007; 17(21):5806-11.

[20] Kim D, Kowalchick JE, Edmondson SD, Mastracchio A, Xu J, Eiermann GJ et al. Triazolopiperazine-amides as dipeptidyl peptidase IV inhibitors: close analogs of JANUVIA (sitagliptin phosphate). Bioorg Med Chem Lett. 2007; 17: 3373-3377.
[21] Laskowski RA, Rullmann JA, MacArthur MW, Kaptein R, Thornton JM. AQUA and PROCHECK-NMR: Programs for checking the quality of protein structures solved by NMR. J. Biomol. NMR. 1996; 8: 477-486.

[22] Lima AH, Souza PRM, Alencar N, Lameira J, Govender T, Kruger HG, Maguire GEM, Alves CN. Molecular modeling of $T$. rangeli, $T$. brucei gambiense, and $T$. evansi Sialidases in complex with the DANA inhibitor. Chem Biol Drug Des. 2012; 80: 114-120.

[23] Lovell SC, Davis IW, Arendall WB III, Bakker de PIW, Word JM, Prisant MG, Richardson JS, Richardson DC. Structure validation by Calpha geometry: phi, psi and Cbeta deviation. Proteins: Structure, Function \& Genetics. 2002; 50: 437-450.

[24] Melo F, Devos D, Depiereux E, Feytmans. ANOLEA: a www server to assess protein structures. Proc. Int. Conf. Intell. Syst. Mol. Biol. 1997; 5: 187-190.

[25] Mizun CS, Chittiboyina AG, Kurtz TW, Pershadsingh HA, Avery MA. Type 2 diabetes and oral anti-hyperglycemic drugs. Curr Med Chem. 2008; 15:61-74.

[26] Morris AL, MacArthur MW, Hutchinson EG, Thornton JM. Stereochemical quality of protein structure coordinates. Proteins. 1992; 12: 345-364.

[27] Morris GM, Lim-Wilby M. Molecular Docking, Methods in Molecular Biology. In: Molecular Modeling of Proteins, Kukol, A. (Ed.). Humana Press, Totowa, New Jersey, 2008; pp: 365-382.

[28] Mustard D, Ritchie DW. Docking essential Dynamics Eigenstructures. Proteins: Struct Funct Bioinfo. 2005, 60(2) 269-274.

[29] Nongonierma AB, FitzGerald RJ. An in silico model to predict the potential of dietary proteins as sources of dipeptidyl peptidase IV (DPP-IV) inhibitory peptides. Food Chemistry. 2014; 165: 489-498.

[30] Nukala UA, P. Sahithi and P. Raja Rao. In-silico Structure based, QSAR and Analogue based Studies using Dipeptidyl Peptidase 4 (DPP4) Inhibitors against Diabetes Type-2. Int J. of Biote \& Bio Sci. 2015; 1 (1): 16-20.

[31] Prajapat R, Bhattacharya I. In Silico Structure Analysis of Type 2 Diabetes Associated Cysteine Protease Calpain-10 (CAPN10). Adv in Diabetes and Metabolism. 2016; 4: 32-43. doi: 10.13189/adm.2016.040202.

[32] Prajapat R, Marwal A, Gaur RK. Recognition of Errors in the Refinement and validation of three-dimensional structures of $\mathrm{AC} 1$ proteins of begomovirus strains by using ProSA-Web. J. of Viruses. 2014; ID 752656, 6 pages.doi.org/10.1155/2014/ 752656 .

[33] Ritchie DW, Grudinin S. Spherical Polar Fourier Assembly of Protein Complexes with Arbitrary Point Group Symmetry. J. of Applied Crystallography. 2016; 49(1), 158-167.

[34] Ritchie DW, Kozakov D, Vajda S. Accelerating protein-protein docking correlations using a six-dimensional analytic FFT generating function. Bioinformatics, 2008; 24: $1865-1873$

[35] Ritchie DW, Venkatraman V. Ultra-Fast FFT Protein Docking On Graphics Processors. Bioinformatics, 2010; 26, 2398-2405.

[36] Sebokova E, Christ AD, Boehringer M, Mizrahi J.Dipeptidyl 
peptidase IV inhibitors: the next generation of new promising therapies for the management of type 2 diabetes. Curr Top Med Chem. 2007; 7:547-555.

[37] Sicree R, Shaw J, Zimmet P. Diabetes and impaired glucose tolerance. Diabetes Atlas. International Diabetes Federation. $3^{\text {rd }}$ ed. Belgium: International Diabetes Federation. 2006; 15-103.

[38] Tabish SA. Is Diabetes Becoming the Biggest Epidemic of the Twenty-first Century? Int. J. of Health Sci. 2007; 1(2), V-VIII.

[39] Teilum K, Hoch JC, Goffin V, Kinet S, Martial JA, Kragelund BB. Solution structure of human prolactin. J. of Mol Bio. 2005; 351(4):810-823.

[40] Uma AN, Sahithi P, Rao PR. In-silico Structure based, QSAR and Analogue based Studies using Dipeptidyl Peptidase 4 (DPP4) Inhibitors against Diabetes Type-2. Int. J. of Biotech. and Biomed. Sci. 2015, 1(1): 16-20.

[41] Wb AE. Dipeptidyl peptidase IV inhibitors for the treatment of diabetes. J. Med Chem, 2004; 47: 4135-4141.

[42] Wiederstein M, Sippl MJ. ProSA-web: interactive web service for the recognition of errors in three-dimensional structures of proteins. Nucleic Acids Research. 2007; 35: 407-410.

[43] Zander M, Madsbad S, Madsen JL, Holst JJ. Effect of 6-week course of glucagon-like peptide 1 on glycaemic control, insulin sensitivity, and $\beta$-cell function in type 2 diabetes: a parallel-group study. Lancet. 2002; 359:824-830.

[44] Zhang Z, Schwartz S, Wagner L, Miller W. A greedy algorithm for aligning DNA sequences. J. Comput Biol. 2000; 7: 203-214.

[45] Zhaolan L, Chaowei F, Weibing W, Biao X. Prevalence of chronic complications of type 2 diabetes mellitus in outpatients-a correstional hospital based survey in urban China. HQLO. 2010; 8: 62-71.

[46] Zimmet P, Alberti KG, Shaw J. Global and societal implications of the diabetes epidemic. Nature. 2001, 414:782-7. 